\title{
Studies on Piezo-Magnetization (I)
}

\section{Magnetization of Titaniferous Magnetite under Uniaxial Compression*}

\author{
By Takesi Nagata and Hajimu Kinoshita \\ Geophysical Institute, University of Tokyo, Tokyo. \\ (Read May 13, 1963 and May 27, 1964 ; Received April 15, 1965)
}

\begin{abstract}
Changes in the magnetization curves, magnetic susceptibility, and remanent magnetization of magnetite and titaniferous magnetite under uniaxial compression are experimentally examined in a systematic way. The uniaxial compression results in a reduction of the intensity of magnetization and the magnetic susceptibility of both single crystals and assemblages of fine grains of titaniferous magnetite.

However, a removal of the compression from the samples in presence of a magnetic field results in an abrupt increase of magnetization, the resultant intensity of magnetization being larger than the ordinary magnetization without the effect of compression in the same field. The majority of the increased magnetization remains as the remanent magnetization after removal of the field. Thus, the isothermal remanent magnetization produced by removing uniaxial compression in a magnetic field increases with increasing magnitude of the uniaxial compression.
\end{abstract}

\section{Introduction}

Magnetization of the earth's crust may be appreciably changed by mechanical stress. Extensive experimental studies on the change in magnetic susceptibility caused by uniaxial compression were made by Kalashnikov and Kapitsa (1952) on rocks and by Grabovsky and Parkhomenko (1953) on magnetites and titaniferous magnetites. Their major result was that the magnetic susceptibility $(\chi)$ decreases hyperbolically with increase in the uniaxial compression $(P)$ in a form of $\chi(P)=\chi_{0} /(1+\beta P)$. This result has been interpreted by Kern (1961) and Stacey (1962) as due to an increase in the magnetoelastic energy which acts as the restorage force against the magnetic field energy produced by the applied magnetic field.

On the other hand, Domen (1957) showed experimentally that a coagulated assemblage of magnetite grains can acquire an additional remanent magnetization by uniaxial compression in a magnetic field. The intensity of the remanence, after releasing the sample from both the compression and the magnetic field, increases with increase in the applied compression. Summarizing all these results, one may state that the reversible magnetization under a uniaxial compression decreases, and on the contrary, the remanent magnetization after the release of the compression increases with increase in the compression.

* Contribution from Division of Geomagnetism and Planetary Physics, University of Tokyo, Series II, No. 187. 
Therefore, the effect of uniaxial compression on magnetization has been by no means clarified yet.

The present series of experimental works takes aim to clarify the above-mentioned apparent contradiction and to get a consistent and systematic knowledge of the effect of mechanical stress upon magnetization of rocks. We may call here the magnetization caused specifically by mechanical stress piezo-magnetization in general.

\section{Experimental procedures}

The equipment to measure the magnetization under uniaxial compression is a ballistic magnetometer, as schematically illustrated in Fig. 1. As the sample to be measured is fixed between two pieces of non-magnetic anvil, the moving search coil method is inevitably

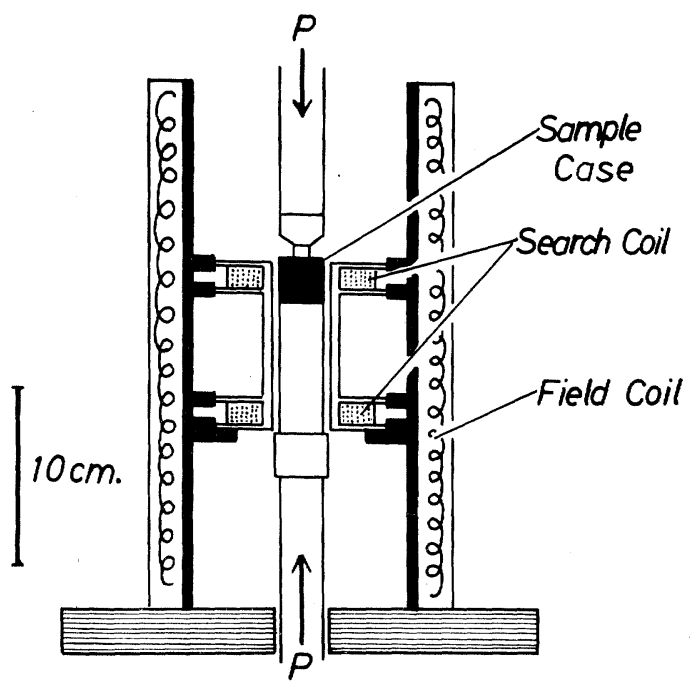

Fig. 1 Schematic view of a ballistic magnetometer for measuring magnetization under uniaxial compression.

adopted. As shown in Fig. 1, two search coils reversely connected are fixed to the frame of the solenoid field coil, and the whole coil system having a counter balance weight can be smoothly and rapidly moved with respect to the fixed sample. Other details of the ballistic magnetometer system were designed based on the ordinary principle of the method (Nagata, 1953, 1961). The main parts of the used uniaxial press are made of stainless steel 18-8 and brass, but the anvils which contact with the sample are made of a $\mathrm{Cu}-\mathrm{Be}$ alloy. Some details of the sample holding system are illustrated in Fig. 2. The sample holding mechanism was a little modified in case of measurement of single crystals, as will be described later.

The strength of the uniaxial pressure was measured by means of a Bourdon tube pressure guage. The maximum total force available by the uniaxial press is about 4.5 tons. 


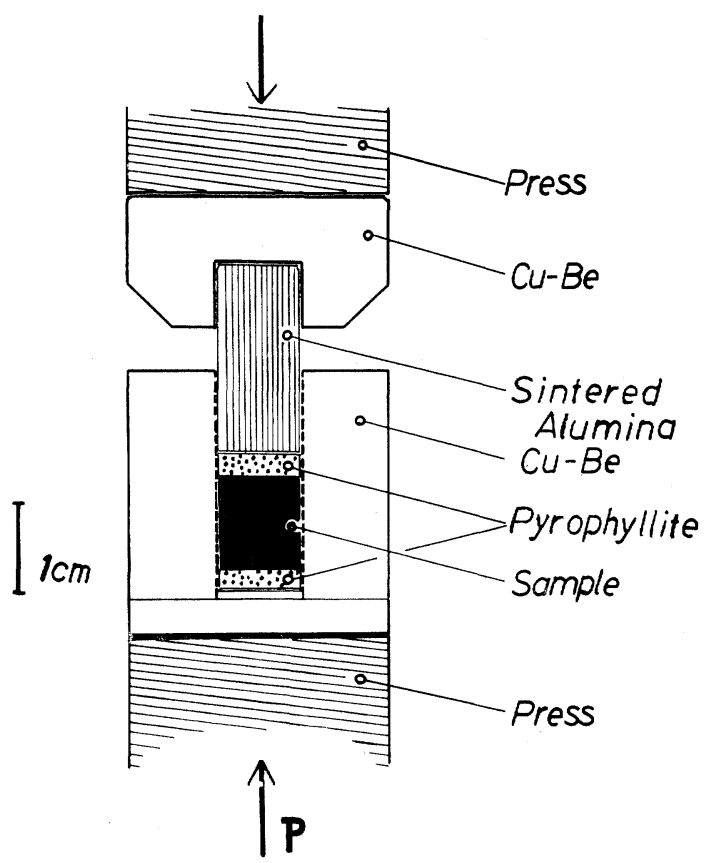

Fig. 2 Structure of sample holder and anvil of the press.

\section{Magnetization curve of single crystal of titaniferous magnetite under uniaxial compression.}

Single crystals of natural titaniferous magnetite (from Mitsuishi, Hokkaido, Japan) were examined as the basic sample. Two circular cylinders of $5 \mathrm{~mm}$ in diameter and about $6 \mathrm{~mm}$ in length are cut out from natural single crystals. The axis of the cylinder is parallel to the [111] direction in one piece and to the [100] direction in the other one. These cylindrical samples were supported by the anvils as illustrated in Fig. 3, where a circular ring of $\mathrm{Cu}-\mathrm{Be}$ alloy surrounds the sample and pyrophyllite is filled in the gap between the sample and the anvils.

The magnetic hysteresis curves for both the [100] axis and [111] axis samples are shown in Figures 4 and 5, where the external magnetic field $H_{e x}$ is parallel to the direction of the

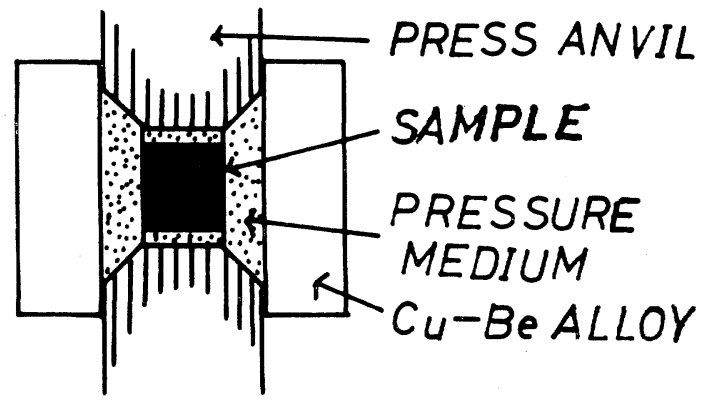

Fig. 3 Sample holder for single crystals. 

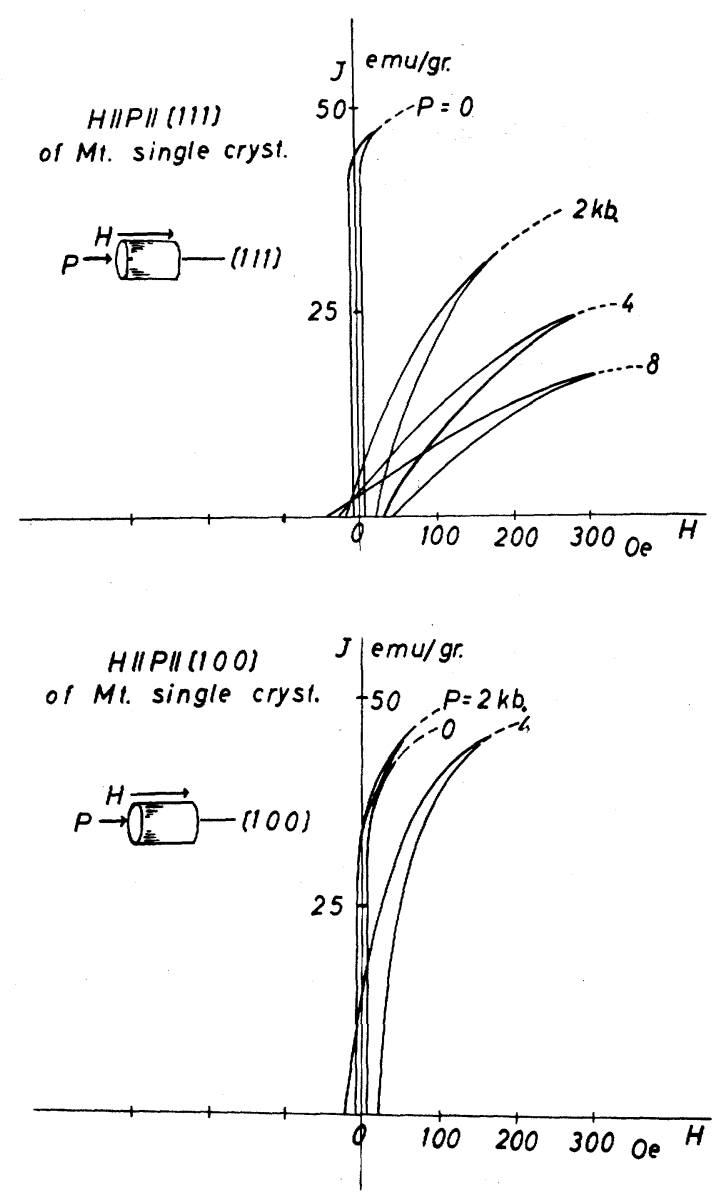

Fig. 4 (Top) Magnetization curves along [111] axis of titaniferous magnetite single crystal under uniaxial compression.

Fig. 5 (Bottom) Magnetization curves along [100] axis of titaniferous magnetite single crystal.

applied uniaxial compression. In general, the effect of the uniaxial compression is much larger along the [111] direction than along the [100] direction. Actually, the uniaxial compression smaller than $2 \mathrm{~kb}$ in strength does not appreciably affect the magnetization curve in case of the [100] axis sample. On the contrary, the uniaxial compression larger than $0.5 \mathrm{~kb}$ in strength appreciably reduce the magnetization of the [111] axis sample.

The dependence of magnetic susceptibility $\chi(P)$ upon the uniaxial compression $P$ is summarized in Fig. 6, where $\chi(P)$ is defined as $\chi(P)=J(P) / H_{e x}$ for the initial linear part of the magnetization curve. The empirical values of $\chi(P) / \chi(P=0)$ are expressed as

$$
\begin{array}{ll}
\chi(P) / \chi(P=0)=1 /(1+0.0075 P)=1-0.0075 P & \text { for the [100] axis, } \\
\chi(P) / \chi(P=0)=1 /(1+0.085 P) & \text { for the [111] axis, }
\end{array}
$$

where $P$ is expressed in unit of $\mathrm{kb}$. The rate of depression of $\chi(P)$ by $P$ is much smaller 


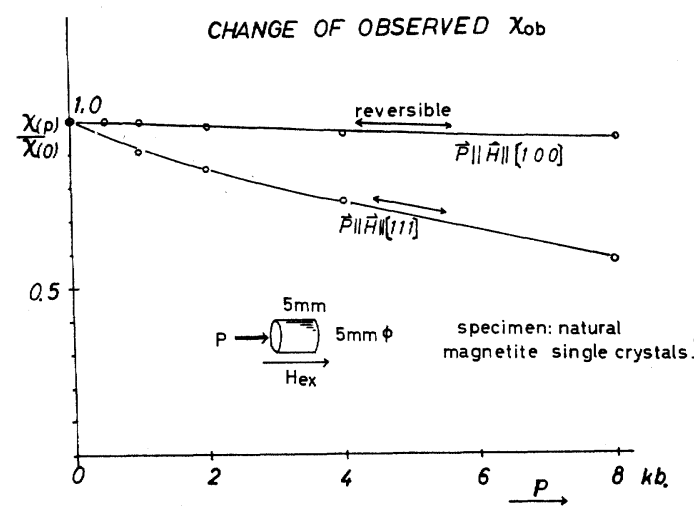

Fig. 6 Magnetic susceptibility of a single crystal of magnetite under uniaxial compression. $\chi_{o b}$ is defined as $J / H_{e x}$, where $J$ is magnetization and $H_{e x}$ is external magnetic field.

along the [100] axis compared with that along the [111] axis.

These effects of the uniaxial compression on magnetization of single crystals of titaniferous magnetites, shown in Figures 4,5 and 6 , are reversible with respect to the compression $P$ within the limit of precision of the present experiments. In the above-mentioned measurements, the magnetic field was applied on the samples which had been kept under the effect of a constant uniaxial compression. In the following series of experiment, the effects of the application and removal of the uniaxial compression on the two single crystals of magnetite, which have already been magnetized in a magnetic field, will be observed.

In Fig. 7, change in magnetization of the samples caused by application of the uniaxial compression $P$ in a magnetic field, $H_{e x}=120 \mathrm{Oe}$, and that caused by removal of the compression in the same field are illustrated, together with the magnetic hysteresis loop of the same samples on the condition of no compression.

In both samples of the [100] and [111] axes, the magnetization in $H_{e x}$ is depressed by applying $P$. The magnitude of decrease becomes larger for larger compression, and it is also definitely larger along the [111] axis than along the [100] axis for the same pressure. By removing the compression from the samples in the same magnetic field, the magnetization jumps up and becomes a little larger than the original magnetization before the compression.

However, the amount of increase of magnetization measured from the original magnetization before the compression is not appreciably dependent on $P$ but reaches nearly the same value. The intensity of remanent magnetization of thus treated samples is larger than that of the ordinary isothermal remanence in each case. The processes of compression and its release in a magnetic field results in an irreversible enhancement of the magnetization of these single crystals. 

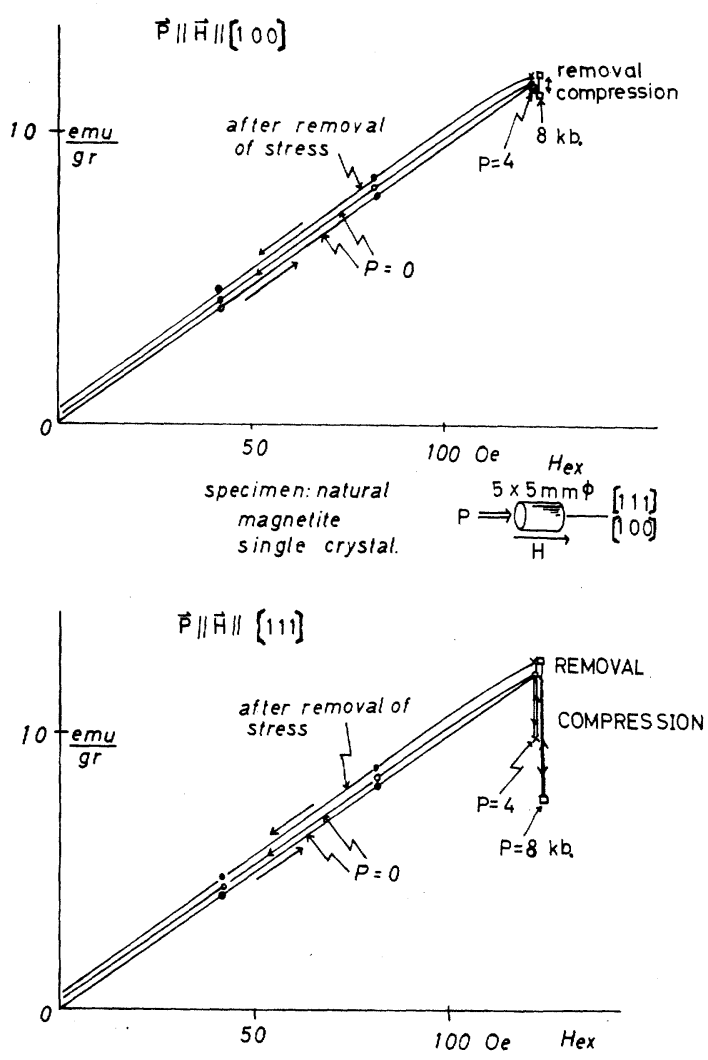

Fig. 7 Effect of application and removal of uniaxial compression upon magnetization of titaniferous magnetite single crystal.

\section{Magnetization curves of assemblage of magnetite grains under uniaxial compression}

The similar effect of uniaxial compression on magnetization of an assemblage of titaniferous magnetite grains (from Niizima biotite rhyolite) was then examined. In case of an assemblage of fine grains, several technical difficulties rise up in the experimental procedures. One of them is that comparatively large grains are apt to be pulverized into smaller grains by the compression. However, repeated processes of compression can stabilize the grain size of such a specimen. An assemblage of titaniferous magnetite grains smaller than mesh 230 of the Tyler standard sieve, obtained finally by the repeated compression processes, seems to be almost stable at least for the range of compression from 0 to $10 \mathrm{~kb}$.

Another problem is a slight deformation of a specimen composed of a large number of fine grains by the compression. This effect can be eliminated also by repeated compressions. The process of $3 \sim 4$ times compression is sufficient to stabilize a specimen composed of the mesh 230 titaniferous magnetite grains. In the following experiments, specimens of grain size of mesh 230 only are used. The specimens of the form as shown in Fig. 2 are compressed up to $4 \mathrm{~kb}$ ten times in a non-magnetic space and then demagnetized by the alternating field method. The samples thus treated are considered as the initial non-magnetized sample. The 
dimensions of each sample were measured after the final compression.

Fig. 8 illustrates the magnetization curves of thus treated sample for 0,2 and $4 \mathrm{~kb}$ of the uniaxial compression, the direction of which is parallel to that of the applied magnetic field. As shown in the figure, the magnetization, the magnetic susceptibility and the remanent magnetization decrease with increase of the uniaxial compression. The coercive force, on the contrary, increases with increase of the compression.

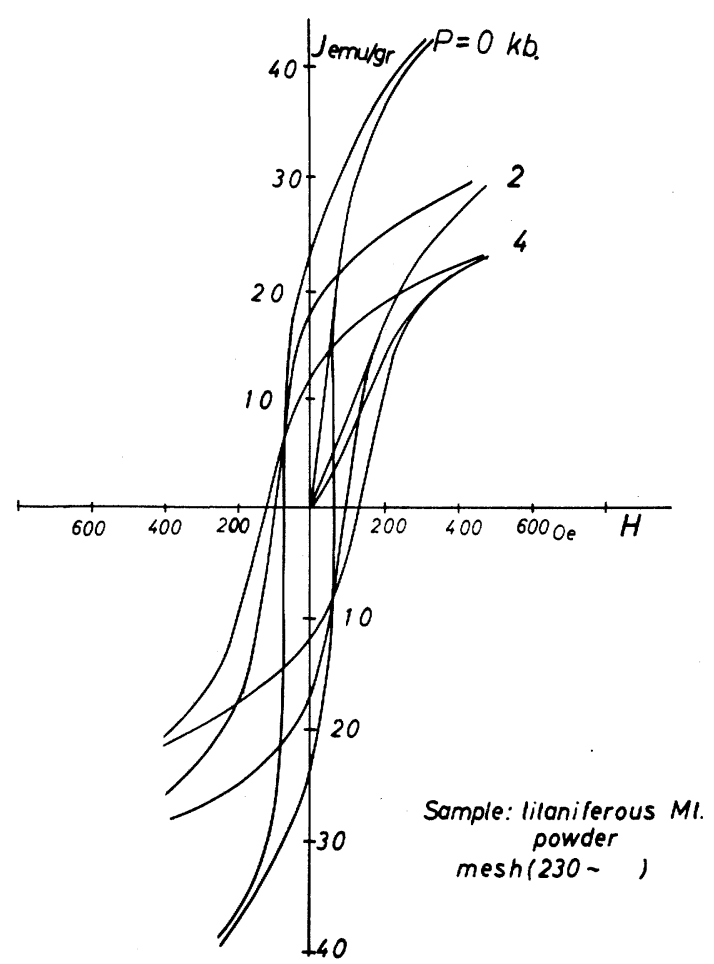

Fig. 8 Magnetization curvs of an assemblage of titaniteraus magnetite grains under uniaxial compression.

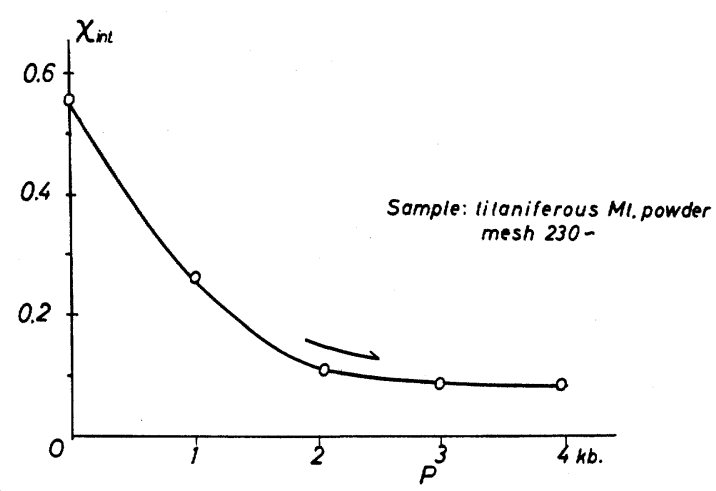

Fig. 9 Dependence of magnetic susceptibility of an assemblage of magnetite grains upon uniaxail compression. 
The dependence of magnetic susceptibility $\chi(P)$ of the sample on the uniaxial compression $P$ is shown in Fig. 9. The value of $\chi(P)$ decreases with $P$ approximately in a hyperbolic form as $\chi(P) / \chi(P=0)=1 /(1+\beta P)$. However, the rate of decrease of $\chi(P)$, or in other words, the magnitude of $\beta$ (about $1.6 / \mathrm{kb}$ ) in this case extremely large compared with the case of single crystal. The large value of $\beta$ of similar magnitude was observed also in Grabovsky and Parkhomenko's measurements (1953) on assemblage of magnetite grains. It may be presumed therefore that the magnetic susceptibility of magnetite grains.is more sensitive to the uniaxial compression than that of the single crystal of large size.

As Kern (1961) suggested theoretically, the effect of compression on the magnetic susceptibility of magnetite can amount approximately to the observed order of magnitude in the present case, if the magnetocrystalline anisotropy is ignored and therefore the magnetization is subject only to the stress-induced anisotropy, or in other words, if change in the magnetization is attributable to rotation of the spontaneous magnetization of individual domains.

In this report we denote by $H_{+}$and $H_{0}$ respectively operations of applying and removing a magnetic field applied onto a specimen, by $H \uparrow$ and $H \downarrow$ respectively operations of increasing and decreasing a magnetic field intensity applied on a specimen and by $P_{+}$and $P_{0}$ respectively uniaxial compression applied and removed from a specimen. In all cases below we also denote the order of operation, applying and removing of a magnetic field $H$ and uniaxial compression $P$, by ordering of signs such as $\left(H_{+}, H_{0}\right.$, etc.) in the parenthesis. For instance, isothermal remanent magnetization IRM will be denoted with subscript as $J_{r}\left(H_{+}, H_{0}\right)$, initial magnetization of uncompressed sample in a magnetic field will be $J\left(H_{+}\right)$and a intensity of initial magnetization in a increasing magnetic field will be Jinit. $(H \uparrow)$. When a uniaxial compression $P$ is applied and removed onto sample after its magnetization, the notation of the operation must be $J\left(H_{+}, P_{+}, P_{0}\right)$.

By this way let us note the initial magnetization in $H$ of a sample compressed by $P$ as Jinit. $\left(P_{+}, H \uparrow\right)$, and the magnetization along the descending curve of its hysteresis loop from the maximum magnetic field $H_{m}$ as $J\left(P_{+}, H_{m+}, H \downarrow\right)$. Then, as mentioned above, the initial magnetization of an uncompressed sample and the descending magnetization of hysteresis loop from $H_{m}$ be denoted by Jinit. $(H \uparrow)$ and $J\left(H_{m+}, H \downarrow\right)$ respectively. The results of the present experiments show that

$$
\begin{aligned}
& \text { Jinit. }\left(H_{\uparrow}\right)>\text { Jinit. }\left(P_{+}, H \uparrow\right) \\
& J\left(H_{m+}, H \downarrow\right)>J\left(P_{+}, H_{m+}, H \downarrow\right), \quad \text { for } \quad H \geqq 0 .
\end{aligned}
$$

Since $J_{r}\left(H_{m+}, H_{0}\right)$ and $J_{r}\left(P_{+}, H_{m+}, H_{0}\right)$ represent the remanent magnetizations of respective cases, we can write

$$
J_{r}\left(H_{m+}, H_{0}\right)>J_{r}\left(P_{+}, H_{m+}, H_{0}\right) .
$$

So long as $H$ is smaller than $H_{m}=80$ Oe in the present case,

Jinit. $(H \uparrow) / H \equiv \chi(0)$, and Jinit. $\left(P_{+}, H \uparrow\right) / H \equiv \chi(P)$.

Therefore 


$$
\chi(0)>\chi(P)
$$

Further,

$$
\frac{1}{J_{r}\left(H_{m+}, H_{0}\right)} \times \frac{\partial J_{r}\left(P_{+}, H_{m+}, H_{0}\right)}{\partial P}<0, \quad \frac{1}{\chi(0)} \times \frac{\partial \chi(P)}{\partial P}<0 .
$$

Let us consider then a case that an initially uncompressed sample is uniaxially compressed by $P$ in $H_{e x}\left(\equiv H_{m}\right)$. The magnetization in this case be denoted by $J\left(H_{m+}, P_{+}\right)$. Fig. 10

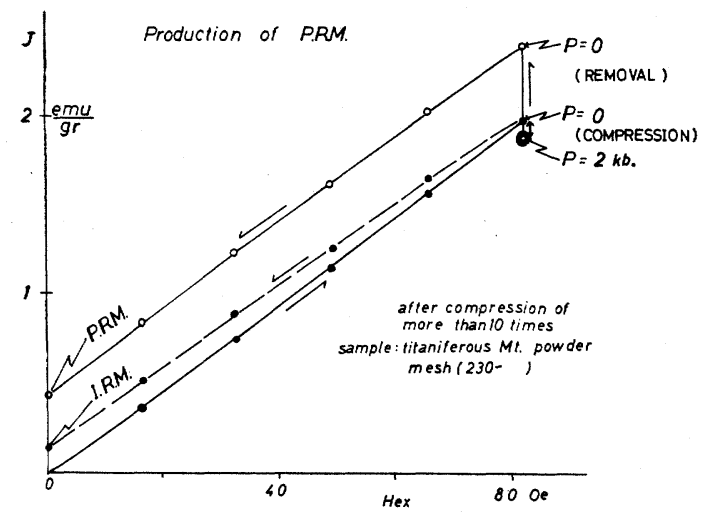

Fig. 10 Effect of application and removal of uniaxail compression on magnetization curve of an assemblage of magnetite grains.

$$
\begin{array}{ll}
\text { : } & J_{\text {init }}(H \uparrow) \text { and } J\left(H_{m+}, H \downarrow\right) \text { curue } \\
\text { : } & J\left(H_{m_{+}}, P_{+}\right) \\
\bigcirc: & J\left(H_{m_{+}}, P_{+}, P_{0}, H \downarrow\right) \text {, (see text.) }
\end{array}
$$

illustrates an example, where the direction of $P=2 \mathrm{~kb}$ is parallel to that of $H$. The magnetization in $H$ is depressed by applying $P$ just as in the case of the single crystal shown in Fig. 7. However, the rate of depression of magnetization by $P$ is smaller in the present case of an assemblage of titaniferous magnetite grains than in the case of the [111] axis single crystal.

Regardless of the rate of depression, we can write, in general,

$$
\frac{1}{J\left(H_{m+}\right)} \times \frac{\partial J\left(H_{m+}, P_{+}\right)}{\partial P}<0 .
$$

When the uniaxial compression is released from the sample in $H$, the magnetization jumps up beyond the original magnetization, $J\left(H_{m+}\right)$, before the compression. Following the above mentioned notation, let us note the magnetization after the removal of the compression in $H$ as $J\left(H_{+}, P_{+}, P_{0}\right)$.

Then

$$
J\left(H_{+}, P_{+}, P_{0}\right)>J\left(H_{+}\right)>J\left(H_{+}, P_{+}\right) .
$$

This inequality relationship holds also in the case of single crystals. In the present case, however,

$$
J\left(H_{+}, P_{+}, P_{0}\right)-J\left(H_{+}\right)>J\left(H_{+}\right)-J\left(H_{+}, P_{+}\right)
$$


Namely, the amount of increase in magnetization caused by the removal of compression is much larger than that of decrease caused by applying the compression. This is a conspicuous contrast to the case of the [111] axis single crystal, for which $J\left(H_{+}, P_{+}, P_{0}\right)-J\left(H_{+}\right)<J\left(H_{+}\right)$ $-J\left(H_{+}, P_{+}\right)$. The magnetization along the descending curve of hysteresis loop from $H_{m}$, at which the uniaxial compression is applied and then removed, be denoted by $J\left(H_{m}+, P+\right.$, $\left.P_{0}, H \downarrow\right)$. Then

$$
J\left(H_{m+}, P_{+}, P_{0}, H \downarrow\right)>J\left(H_{m+}, H \downarrow\right)
$$

and consequently

$$
J_{r}\left(H_{m+}, P_{+}, P_{0}, H_{0}\right)>J_{r}\left(H_{m+}, H_{0}\right) .
$$

Namely, a comparatively large irreversible magnetization is acquired by removing $P$ in $H=H_{m}$, and it remains as a comparatively large remanent magnetization, where $H_{m}$ is about 100 Oe in these experiments.

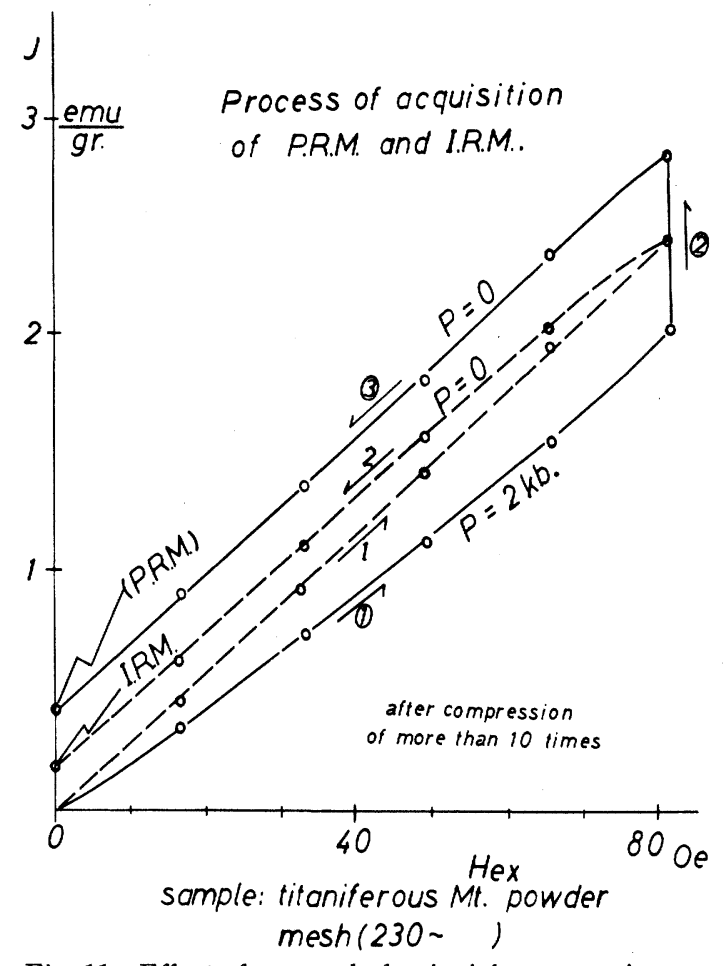

Fig. 11 Effect of removal of uniaxial compression on magnetization curve of an assemblage of magnetite grains magnetized under the compression in comparison with magnetization curve obtained under compression-free condition.

Process 1-2 denotes $\operatorname{Jinit}(H \uparrow)$ and $J\left(H_{m+}\right.$, $H \downarrow)$, (1)-(2)-(3) denotes $\operatorname{Jinit}\left(P_{+}, H \uparrow\right)$ and $J\left(P_{+}, H_{+}, P_{0}\right)$ and $J\left(P_{+}, H,_{+}, P_{0}, H \downarrow\right)$ (see text). (PRM) coincides with the expression $J_{r}\left(P_{+}, H_{m+}, P_{0}, H_{0}\right)$ in the text. 
When $P$ is released from the sample having $J\left(P_{+}, H_{+}\right)$at $H$, the magnetization also jumps up beyond the $J\left(H_{+}\right)$value. Fig. 11 illustrates such an example, where $P=2 \mathrm{~kb}$ and parallel to $H$. The magnetization after the removal of $P$ in $H$ be denoted by $J\left(P_{+}, H_{+}, P_{0}\right)$, then

$$
J\left(P_{+}, H_{+}, P_{0}\right)>J\left(H_{+}\right)>J\left(P_{+}, H_{+}\right) .
$$

The intensity of $J\left(P_{+}, H_{+}, P_{0}\right)$ thus acquired is approximately equal to or a little smaller than that of $J\left(H_{+}, P_{+}, P_{0}\right)$ for the same values of $H$ and $P$, namely

$$
J\left(P_{+}, H_{+}, P_{0}\right) \simeq J\left(H_{+}, P_{+}, P_{0}\right) .
$$

Comparing Fig. 10 with Fig. 11, it may be seen that

$$
J\left(H_{+}\right)-J\left(P_{+}, H_{+}\right)>J\left(H_{+}\right)-J\left(H_{+}, P_{+}\right) .
$$

The magnetization $J\left(P_{+}, H_{m+}, P_{0}, H \downarrow\right)$ along the descending curve from $H_{m}$ at which $P$ is removed in this case is also larger than $J\left(H_{m+}, H \downarrow\right)$, i.e.,

and

$$
J\left(P_{+}, H_{m+}, P_{0}, H \downarrow\right)>J\left(H_{m+}, H \downarrow\right),
$$

$$
J_{r}\left(P_{+}, H_{m+}, P_{0}, H_{0}\right)>J_{r}\left(H_{m+}, H_{0}\right)
$$

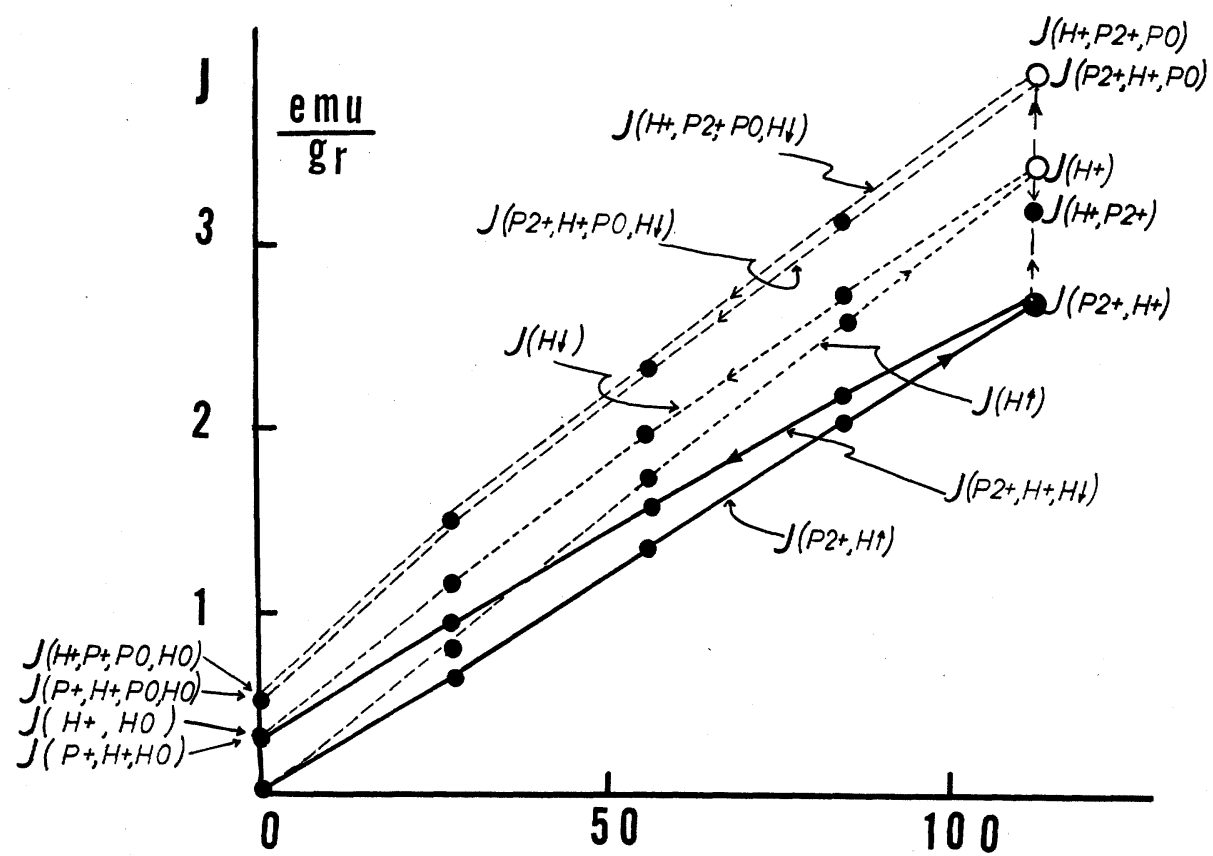

Fig. 12 Magnetization curves of titaniferous magnetite grain assemblage. $J(H \uparrow)$, magnetization with incresing $H$ in $P=O ; J(H \downarrow)$, magnetization with decresing $H$ in $P=O$; $J\left(P_{2+},-H \uparrow\right)$, magnetization with increasing $H$ in $P=2 k b ; J\left(P_{2+}, \mathrm{H}_{+}, H \downarrow\right)$, magnetization with decreasing $H$ in $P=2 k b ; J\left(H_{+}, P_{2+}, P_{0}, H \downarrow\right)$, magnetization] with decresing $H$ after releasing of $P$ from $J\left(H_{+}, P_{2+}\right)$ in $H(\equiv H \mathrm{~m})=112 O \mathrm{e} ; J\left(P_{2+}{ }^{{ }^{*}} H_{+}, P_{0},{ }^{\top} H \downarrow\right)$, magnetization with decreasing $H$ after releasing of $P$ from $J\left(P_{2+}, H_{+},\right)$in $H=112$ $O$ e $J\left(H_{+}, P_{2+}\right)$, magnetization after compressing of $J\left(H_{+}\right)$in $H=112 \mathrm{Oe} ;\left(P_{2^{+}}\right)$, pressure in $2 k b$ applied onto a specimen. 
Fig. 12 illustrates the $J\left(H_{m+}, P_{+}, P_{0}, H \downarrow\right)$ and $J\left(P_{+}, H_{m+}, P_{0}, H \downarrow\right)$ curves in comparosin with Jinit. $\left(P_{+}, H \uparrow\right)$ and $J\left(P_{+}, H_{m+}, H \downarrow\right)$ curves of the same sample. In this case the defined piezoremanent-magnetization PRM is expressed as $J_{r}\left(H_{+}, P_{+}, P_{0}, H_{0}\right)$. Other details are described in Fig. 12.

In case of the sample of titaniferous magnetite grain assemblage, measured values of magnetization are a little inaccurate, probably owing to the still remaining irreversible effect of crushing of grains and viscous deformation. Qualitatively speaking, however, there is no room for doubt that the removal of the uniaxial compression in a magnetic field causes a marked increase of magnetization, which exceeds the amount of decrease of magnetization caused by the compression, and that the major part of irreversible magnetization thus acquired remain as the remanent magnetization after the applied magnetic field is removed.

From the experimental results described above it has been concluded that

$$
J_{r}\left(H_{+}, P_{+}, P_{0}, H_{0}\right) \simeq J_{r}\left(P_{+}, H_{+}, P_{0}, H_{0}\right)>J_{r}\left(H_{+}, H_{0}\right) \simeq J_{r}\left(P_{+}, H_{+}, H_{0}\right)
$$

It may thus be clear that the remanent magnetization of magnetite subjected to both the uniaxial compression and a magnetic field takes different values for different orders of application and removal of the compression and a magnetic field.

Measurements of the remanent magnetization of the titaniferous magnetite grain samples are easily and much precisely carried out by means of an ordinary magnetometer. Fig. 13 summarizes the results of measurements of the remanent magnetization acquired by the

$$
P \| \text { Hex }=50 \text { oe }
$$
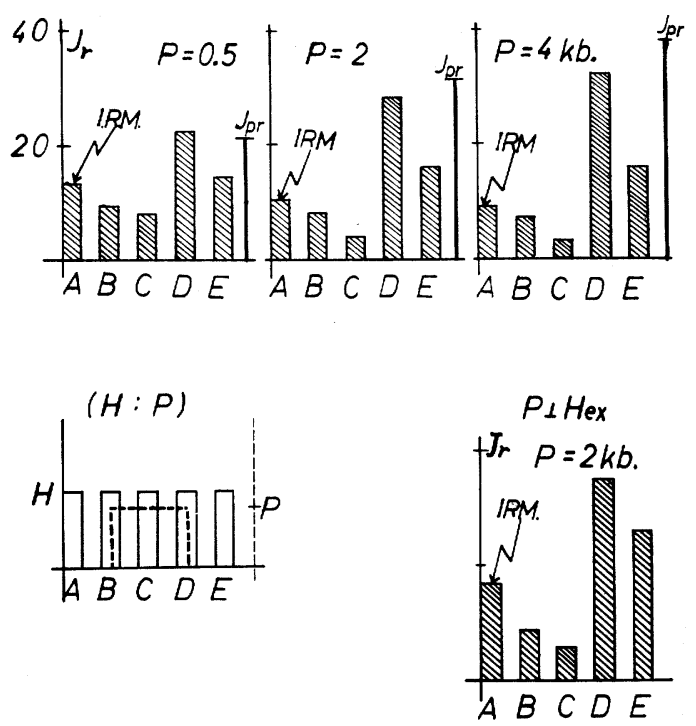

Fig. 13 Remanent magnetization of magnetite powder after applying a magnetic field $(H)$ and uniaxial compression $(P)$ in different mutual orders (indicated on the left side down).
A : $J_{r}\left(H_{+}, H_{0}\right)$
B: $J_{r}\left(H_{+}, P_{+}, H_{0}, P_{0}\right)$
$\mathrm{C}: J_{r}\left(P_{+}, H_{+}, H_{0}, P_{0}\right)$
$\mathrm{D}: J_{r}\left(P_{+}, H_{+}, P_{0}, H_{0}\right)$
$\mathrm{E}: J_{r}\left(P_{+}, P_{0}, H_{+}, H_{0}\right) \quad J_{p_{r}}: J_{r}\left(H_{+}, P_{+}, P_{0}, H_{0}\right)$ 
six different processes. In case that $P$ is applied parallel to $H$, the remanence was examined for three conditions, $P=0.5,2$ and $4 \mathrm{~kb}$. A case that $P$ is perpendicular to $H$ and $P=2 \mathrm{~kb}$ was also examined.

From the diagrams shown in Fig. 13, it may be generally concluded that

$$
\begin{aligned}
& J_{r}\left(P_{+}, H_{+}, P_{0}, H_{0}\right)>J_{r}\left(P_{+}, P_{0}, H_{+}, H_{0}\right)>J_{r}\left(H_{+}, H_{0}\right)>J_{r}\left(H_{+}, P_{+}, H_{0}, P_{0}\right) \\
& >J_{r}\left(P_{+}, H_{+}, H_{0}, P_{0}\right)
\end{aligned}
$$

and that $J_{r}\left(H_{+}, P_{+}, P_{0}, H_{0}\right)$ and $J_{r}\left(P_{+}, H_{+}, P_{0}, H_{0}\right)$ have exclusively large values compared with all the others. For $P=0.5 \mathrm{kbar}$ in $P / / H_{e x}, J_{r}\left(H_{+}, P_{+}, P_{0}, H_{0}\right)<J_{r}\left(P_{+}, H_{+}, P_{0}, H_{0}\right)$ but for $P=2$ and $4 \mathrm{~kb}$ in $P / / H_{e x}, J_{r}\left(H_{+}, P_{+}, P_{0}, H_{0}\right)>J_{r}\left(P_{+}, H_{+}, P_{0}, H_{0}\right)$. This reversal of the inequality relation may suggest a fairly complicated mechanism of the binary effect of the compression and magnetic field in the sample.

It will be phenomenologically certain, however, that the removal of the uniaxial compression in a magnetic field causes a remarkable increase of remanence as compared with the ordinary isothermal remanence $J_{r}\left(H_{+}, H_{0}\right)$, while the removal of a magnetic field in existence of the uniaxial compression results in a depression of the remanence. In other words, the stage of depressed magnetization under the compression remains as the remanence when the magnetostatic energy caused by a field is removed first and then magnetoelastic energy is finally taken out, but a removal of the compression in existence of a magnetic field causes an irreversible advance of magnetization which much exceeds the amount of depression of irreversible magnetization caused by the compression.

\section{Some qualitative discussions of the observed effects of uniaxial compression on magnetization.}

As shown in Fig. 7, the effect of uniaxial compression on magnetization of magnetite single crystal is mostly reversible with respect to the compression, although removal of the compression in presence of a magnetic field causes an additional magnetization of a small amount which remains as an additional remanent magnetization after the removal of the compression.

On the contrary, the magnitude of irreversible magnetization acquired by removal of the uniaxial compression in presence of a magnetic field is extremely large in case of an assemblage of fine grains, as illustrated in Figures 10.

The above-mentioned observed results may indicate that the main factor of magnetic coercivity of a single crystal of magnetite under compression is due to the magnetocrystalline anisotropy energy and the reversible effect of uniaxial compression may affect the magnetized state through the additional reversible energy of magnetoelasticity. On the other hand, the effect of local anisotropy energy induced by locally accumulated internal stress and the shape anisotropy of very fine grains may also be dominant in magnetization mechanism of an assemblage of fine grains of magnetite.

According to Syono (1965), the magnetocrystalline anisotropy constants, $K_{1}$ and $K_{2}$, and the saturation magnetostriction coefficients, $\lambda_{111}$ and $\lambda_{100}$, of synthesised pure magnetite at 
$290^{\circ} \mathrm{K}$ are

$$
\begin{array}{ll}
K_{1}=-1.36 \times 10^{5} \mathrm{erg} / \mathrm{cm}^{3}, & \lambda_{111}=78 \times 10^{-6} \text { c.g.s. } \\
K_{2}=-0.44 \times 10^{5} \mathrm{erg} / \mathrm{cm}^{3}, & \lambda_{100}=-20 \times 10^{-6} \text { c.g.s. },
\end{array}
$$

and those parameters of magnetite-rich titanomagnetite $0.1 \mathrm{Fe}_{2} \mathrm{TiO}_{4} 0.9 \mathrm{Fe}_{3} \mathrm{O}_{4}$ are given as

$$
\begin{array}{ll}
K_{1}=-2.50 \times 10^{5} \mathrm{erg} / \mathrm{cm}^{3}, & \lambda_{111}=96 \times 10^{-6} \mathrm{c.g} . \mathrm{s} ., \\
K_{2}=0.48 \times 10^{5} \mathrm{erg} / \mathrm{cm}^{3}, & \lambda_{100}=4 \times 10^{-6} \mathrm{c.g} . \mathrm{s} .
\end{array}
$$

The saturation magnetostriction coefficients $\lambda$ s for polycrystalline specimens of $\mathrm{Fe}_{3} \mathrm{O}_{4}$ and $0.1 \mathrm{Fe}_{2} \mathrm{TiO}_{4} 0.9 \mathrm{Fe}_{3} \mathrm{O}_{4}$ are $39 \times 10^{-6}$ and $59 \times 10^{-6}$ respectively. As will be obvious from those values of magnetostriction coefficient, compression of magnetite-rich titanomagnetite results generally in an increase of resistance against magnetization, thus magnetization being suppressed by compression. The effect of hydrostatic compression on the magnetic susceptibility of an assemblage of magnetite grains has been theoretically studied by Kern (1961) to prove that the susceptibility decreases hyperbolically with increasing compression. Theoretical studies on a possible effect of uniaxial compression of a magnetite single crystal, along either the [111] axis or the [100] axis, on its magnetic susceptibility show also that the ratio of susceptibility $\kappa(p)$ under compression $P$ to the normal susceptibility $\kappa_{0}$ without $P$ is expressed as

$$
\kappa(p) / \kappa_{0}=\left\{1+\lambda P f\left(K_{1}, K_{2}\right)\right\}^{-1},
$$

where $\lambda$ represents either $\lambda_{111}$ or $\lambda_{100}$ and $f\left(K_{1}, K_{2}\right)$ is a function of positive value determined only by $K_{1}$ and $K_{2}$. The functional form of $f$ is different in cases of [111] and [100], $f(111)$ being much larger than $f(100)$. Detailed discussions of this theoretical problem will be summarized in the forthcoming report.

As for the irreversible magnetization particularly acquired by removing the uniaxial compression in presence of $H$, the main reason for the case of a single crystal will be as follows. When the direction of $P$ is along the [100] axis, the easy magnetization directions

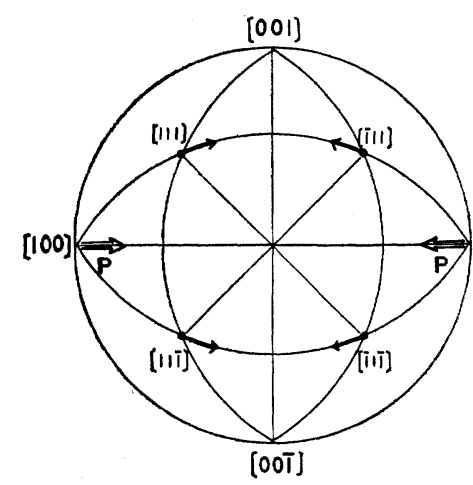

a) Compressed along $[100]-[100]$ axis

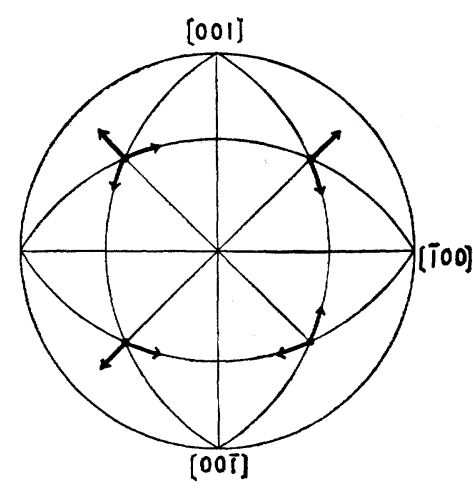

b) Compressed olong [III] - [iii] axis

Fig. 14 Deviation of the easy magnetization directions of magnetite crystal by uniaxial compression. 
which are along the [111] equivalent axes are deviated by $P$ as shown in Fig. 14 (a). When $P$ is along the [111] axis, the easy directions are deviated as shown in Fig. 14 (b). When a magnetic field $H$ is applied onto the direction parallel to $P$, magnetization of a small portion of domains can more easily transfered from the $-H$ side to the $+H$ side over potential barrier of magnetocrystalline energy reduced by the magnetoelastic energy caused by $P$. When $P$ is removed in presence of $H$, the potential barrier between the $-H$ side and the $+H$ side is recovered to its normal value, and consequently the transfered magnetizations hardly come back to their original directions. The irreversible magnetization thus acquired may be identified to the piezo-remanent magnetization of a single crystal of magnetite-rich titanomagnetite.

In case of an assemblage of fine grains, mechanism of acquiring the irreversible magnetization may be more complicated, because of the additional effect of stress-induced snisotropy and shape anisotropy. If we assume, as an extreme case, uniaxial single domain produced by the stress-induced anisotropy owing to local internal stress, the uniaxial compression may result in a rotation of magnetization away from the initial easy direction because $\lambda>0$. If $H$ is strong enough to rotate the magnetization further away towards the opposite side of the initial easy direction, a removal of the uniaxial compression will result in the 180 degree rotation of the magnetization, and the irreversible rotation of magnetization will remain as remanence after $H$ also is removed.

A semi-quantitative examination of the problem along the above-mentioned way seems likely to give a favourable interpretation of the experimental result. These problems will be discussed in more detail together with other experimental data in the forthcoming report.

\section{References}

Domen, H., Bull. Fac. Education Yamaguchi Univ. Japan, 7, 41, 1957.

Grabousky, M.A. and E.I. Parkhomenko, Izvest. Akad. Nauk. USSR, Ser. Geophys. 5, 405, 1953.

Kalashnikov, A.G. and S.P. Kapitsa. Doklady Akad. Nauk, USSR, 86 (3), 521, 1952.

Kern, J.W., J. Geophys. Res., 66, 3807, 1961

Nagata, T. “Rock Magnetism”, 1st ed. Tokyo, 1953, 2nd ed. Tokyo, 1961.

Stacey, F.D., Phil. Mag., 7, 551, 1962.

Syono, Y., Japanese J. Geophys., 4, 71, 1965. 